\title{
Research on application of polynomial fitting technique in rotary kiln
}

\section{infrared temperature measurement system}

\author{
Zhongyuan Guo*, Shaosheng Dai, Jinsong Liu, Changhui You, Yajun Cheng, Liangbing Yu \\ (Chongqing Key Laboratory of Signal and Information Processing, Chongqing University of Posts \\ and Telecommunications, No.2 Chongwen Road, Nan'an District, Chongqing 400065, China) \\ *Corresponding author: guozhongyuan1993@outlook.com; +8615683652580
}

Abstract: Aiming at the linear temperature compensation algorithm's disadvantage of temperature measurement error in rotary kiln infrared scanning temperature measurement process, this paper proposes a precise nonlinear cubic polynomial fitting temperature compensation algorithm. The proposed algorithm compensates the temperature values of scanning points on rotary kiln surface by following steps: Calculating temperature difference between the real temperature value of rotary kiln and temperature value measured by infrared scanning temperature measurement system; Fitting the temperature difference data with cubic polynomial; Using the obtained function to compensate temperature. Experimental result shows that compared with the usual linear temperature compensation algorithm, the accuracy of proposed algorithm has raised about 2.25 times when cubic polynomial is used.

Keywords: rotary kiln, temperature compensation, polynomial fitting, measurement accuracy

\section{Introduction}

Rotary kiln is a thermal equipment for dying and baking materials, it is widely used in the technological process of cement, non-ferrous metallurgy, iron and steel metallurgy and building materials and occupies an important role in the production line of these fields, its running status directly affects production quantity, quality and energy consumption, too high temperature or too large thermal swing may cause kiln lining's damage, even results in "red kiln" accident, so it is necessary to monitor calcination circumstances real time[1]. There are a wide range of temperature monitoring devices of rotary kiln, and among these devices, infrared scanning temperature measurement system (ISTMS) has a wide application in rotary kiln temperature measurement process because of its advantages such as non-contact temperature measurement, high stability and simple installation [2]. However, the scanning distance between rotary kiln and ISTMS affects the temperature measurement accuracy seriously, therefore, it is significant to study the influence of scanning distance on the accuracy of infrared temperature measurement. In the past decades, many experts and scholars at home and board have done a lot of research to reduce the impact of measuring distance on infrared temperature measurement. Chrzanowski[3,4] studied the influence of changes of object-system distance on the temperature measurement accuracy of infrared systems and developed a theory which has shown that the difference between the real measuring distance and calibration distance causes significant temperature measurement errors for high temperature objects. Kargel[5] drawn a conclusion that infrared temperature measurement system could not measure objects' temperature preciously when measuring distance between 
system and object were not determined. Sun [6] analyzed the influence of measuring distance on the temperature measurement accuracy of infrared thermal imager and proposed a method of fitting experimental data to improve temperature measurement accuracy under short-distance condition. Guo[7] analyzed the influence of field angle and measuring distance on the measurement accuracy of infrared systems and introduced an error compensation method. Zhang [8] proposed a method to reduce the influence of measuring distance on measurement accuracy, the effect principle of the contrast between objects' temperature and environmental temperature, the atmospheric transmittance and field angle of infrared system under close range are analyzed. But for ISTMS of rotary kiln, the scanning distance is generally several tens meters and changes constantly during the measurement process, the method for reducing the influence of measuring distance on temperature measurement accuracy should be easy to realize to ensure the real time of data process. The commonly used linear temperature compensation algorithm[9] compensates the temperature value by measuring the maximum distance between rotary kiln and ISTMS and the maximum temperature difference between the real temperature value measured by hand-hold infrared thermometer and the temperature value measured by infrared detector, calculating temperature compensation coefficient and then getting temperature compensation equation, but this linear compensation algorithm is not accordance with infrared radiation attenuation characteristics of ISTMS, so it has large temperature measurement error. This paper proposes a nonlinear polynomial fitting temperature compensation algorithm, experiments show that the proposed algorithm can effectively improve the temperature measurement accuracy.

\section{Infrared scanning temperature measurement principle of rotary kiln}

ISTMS is an optical, mechanical and electronic integration equipment, which combines infrared radiation temperature measurement technology with optical scanning technology[10], it mainly consists of four parts: optical system, motor and driver, infrared detector, signal processor and transmission module, the optical scanning lens driven by motor rotates to collect the infrared radiation on rotary kiln surface and then reflect the radiation to infrared detector, at the same time, rotary kiln rotates on its own axis to complete the overall scanning of rotary kiln surface, as shown in Fig. 1 , then the infrared radiation energy is converted into electrical signal by digital signal processor and then the processor converts the electrical signal into digital temperature signal[11].

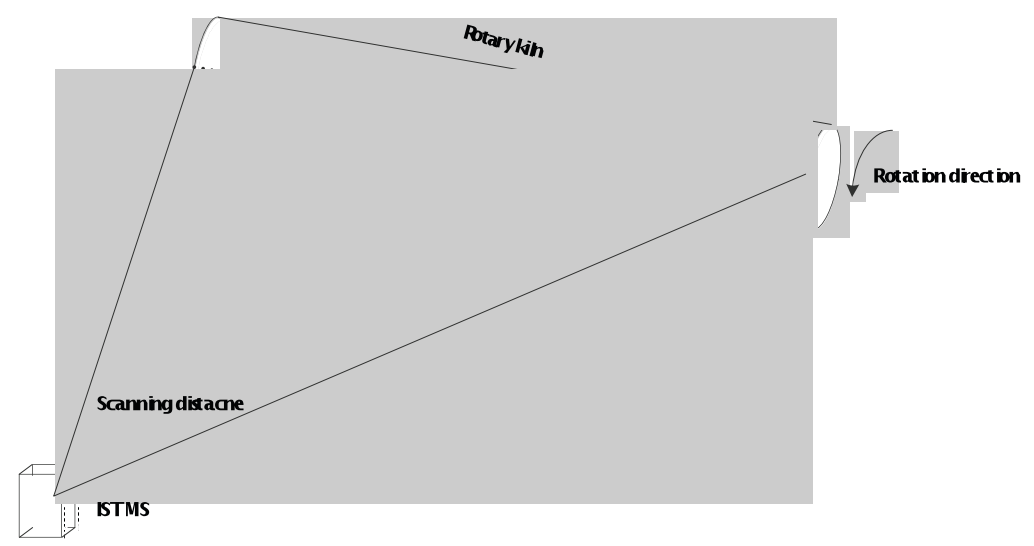


Figure.1 Scanning temperature measurement principle of ISTMS

\section{Linear temperature compensation algorithm}

Linear temperature compensation algorithm $[12,13]$ is widely used in rotary kiln infrared scanning temperature measurement process, the algorithm compensates the temperature value of measuring points on rotary kiln surface by determining linear compensation coefficient and compensating temperature value according to the scanning distance between ISTMS and rotary kiln. The specific implementation process is described as follow:

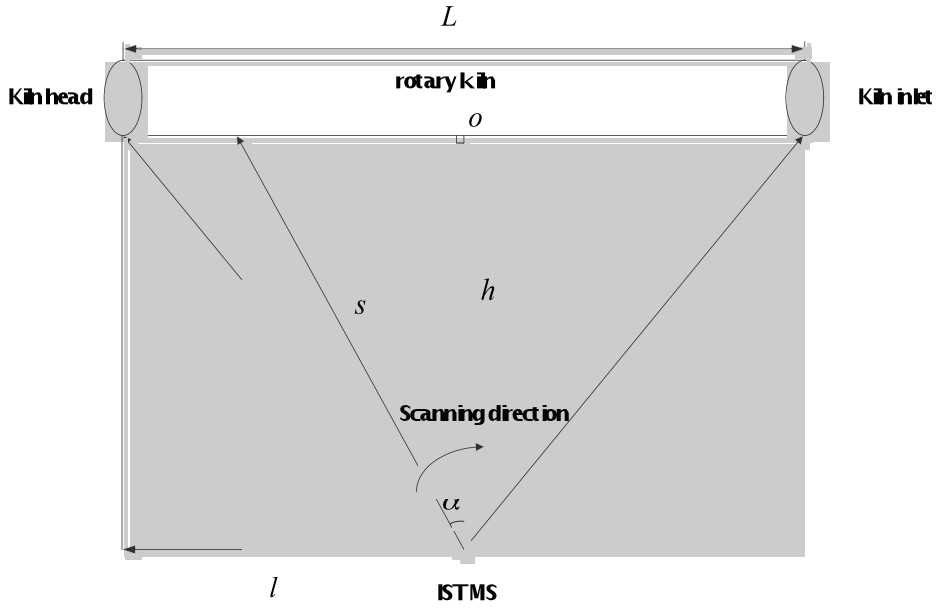

Fig.2.Schematic diagram of traditional temperature compensation algorithm

As shown in Fig.2,where cylindrical object represents rotary kiln, it is supposed that rotary kiln and ISTMS are at the same height, where $O$ represents vertical point between rotary kiln and ISTMS, $L$ represents the length of rotary kiln, $h$ represents the vertical distance between ISTMS and rotary kiln, the distance between kiln head and ISTMS is $l$, the scanning angle is $\alpha$, and $s$ represents the scanning distance, it can be calculated by the following equation:

$$
s=h / \cos \alpha
$$

In order to determine the linear compensation coefficient, the algorithm firstly calibrates the parameters of ISTMS such as the emissivity of rotary kiln and air humidity to ensure that the temperature value of vertical point $O$ measured by ISTMS is in accordance with the real temperature measured by hand-held infrared thermometer in near distance. It is worth mentioning that the temperature values of measuring points on rotary kiln surface measured by hand-held infrared thermometer in near distance can avoid the influences of atmospheric attention and the scanning distance, so it can be regarded as the real temperature value of scanning points on rotary kiln surface. As the coefficient is a fixed value, it can be concluded that when the scanning distance reaches the maximum value, the temperature difference reaches the maximum value.

The calculation method of the maximum scanning distance and the maximum temperature difference can be divided into the following two ways:

1 .When the vertical point is on the left side of midpoint of rotary kiln axis:

$$
\Delta T_{\max }=T_{r t}-T_{i t}
$$


Where $\Delta T_{\max }$ represents maximum temperature difference, $T_{r t}$ represents the real temperature value on rotary kiln tail measured by hand-held infrared thermometer in near distance, $T_{i t}$ represents the temperature value on rotary kiln tail measured by ISTMS without compensation.

At the same time, the maximum scanning distance difference can be expressed as below:

$$
\Delta d_{\max }=s_{\max }-h=\sqrt{(L-l)^{2}+h^{2}}-h
$$

So the equation of temperature compensation coefficient is as follow:

$$
T_{0}=\Delta T_{\max } / \Delta d_{\max }=\left(T_{r t}-T_{i t}\right) /\left[\sqrt{(L-l)^{2}+h^{2}}-h\right]
$$

The expression of temperature compensation can be calculated as follow:

$$
\Delta T=(s-h) \cdot\left(T_{r t}-T_{i t}\right) /\left[\sqrt{(L-l)^{2}+h^{2}}-h\right]
$$

2. When the vertical point is coincidence with the midpoint or on the right side of midpoint of rotary kiln axis:

$$
\Delta T_{\max }=T_{r h}-T_{i h}
$$

Where $T_{r h}$ represents the real temperature value on rotary kiln head, $T_{i h}$ represents the temperature value on rotary kiln head measured by ISTMS without compensation.

$$
\Delta d_{\max }=\sqrt{l^{2}+h^{2}}-h
$$

In this condition, the calculation methods of temperature compensation coefficient and temperature compensation algorithm are described as below:

$$
\begin{gathered}
T_{0}=\left(T_{r h}-T_{i h}\right) /\left[\sqrt{l^{2}+h^{2}}-h\right] \\
\Delta T=(s-h) \cdot\left(T_{r h}-T_{i h}\right) /\left(\sqrt{l^{2}+h^{2}}-h\right)
\end{gathered}
$$

Then temperature value of scanning points on rotary kiln surface is calculated as below:

$$
T=T_{i}+\Delta T
$$

Where $T_{i}$ represent temperature values measured by ISTMS without compensation.

\section{Proposed nonlinear temperature compensation algorithm}

Although linear temperature compensation algorithm is simple to use, there is a big temperature measurement error. In fact, there is a nonlinear relationship between distance and temperature of measured object under remote temperature measurement circumstances [14], so this paper explores a nonlinear temperature compensation algorithm which is more accords with the temperature measurement of rotary kiln to 
improve temperature measurement accuracy.

The atmospheric transmittance decreases with the increase of scanning distance between rotary kiln and ISTMS, which leads to the error of temperature measurement precision, and in a certain range, the difference between the real temperature and the temperature measured by ISTMS increases with the increase of the distance, but when beyond this range, the temperature difference will tend to be stable, which is in line with polynomial function.

Based on the above conclusion, a nonlinear temperature compensation algorithm is proposed to improve rotary kiln temperature measurement accuracy, the specific implementation process are as follows:

In order to determine temperature compensation formula, experiments use an independent researched and developed ISTMS designed by Chongqing Key Laboratory of Signal and Information Processing, it is worth noting that this series of products have been widely used in cement production site, achieving satisfactory results. In addition, a black body radiator is used as measured object, whose emissivity is 0.98 .As the experiments are carried out in closed indoor environment, the surrounding temperature and humidity in a certain time are relatively stable, and other influencing factors of temperature measurement can be neglected. In order to simulate the actual application scenarios, the scanning distance data between ISTMS and black body radiator are set from 30 meters to 50 meters per unit distance, and the temperature value of black body radiator is set as $307^{\circ} \mathrm{C}$, where 30 meters is used as calibration point, representing that the temperature value measured by ISTMS is consistent with that of black body. The temperature data are collected and curved, as shown in Fig. 3.

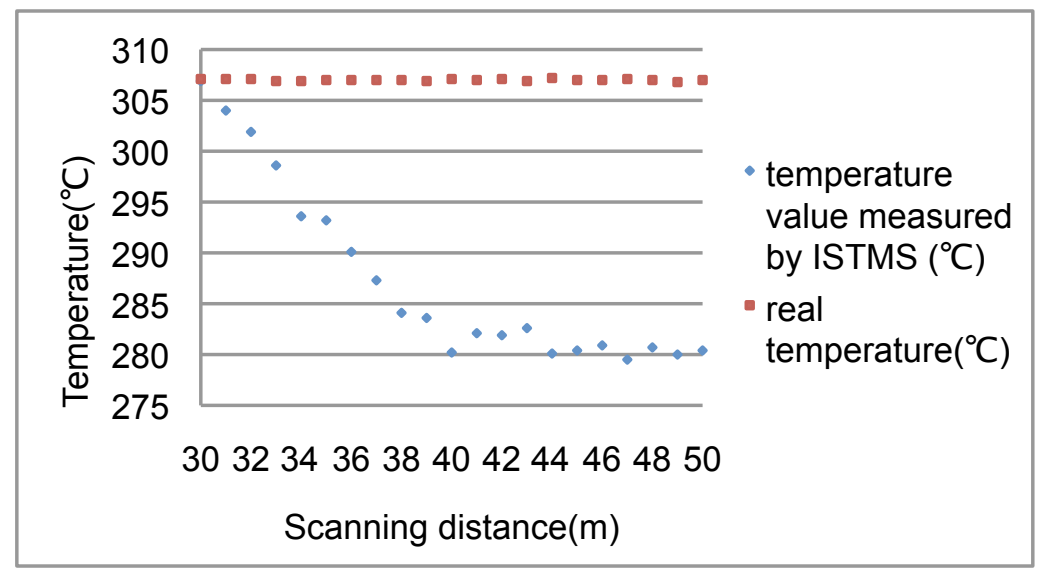

Figure. 3 The temperature of real temperature and the temperature measured by ISTMS at different scanning distance

The temperature difference between the real temperature displayed by black body radiator and the temperature value measured by ISTMS are calculated to further analyze temperature compensation formula, and cubic polynomial fitting is used to show the relationship between scanning distance and temperature difference, whose equation form is shown as below :

$$
\Delta T=a \cdot s^{3}+b \cdot s^{2}+c \cdot s+d
$$


Where $\Delta T$ represents the temperature difference between the real temperature and the temperature value measured by ISTMS, $s$ represents scanning distance between rotary kiln and ISTMS, $a, b, c, d$ are constants, which can be drawn by programming, the fitting curve is shown in Fig.4:

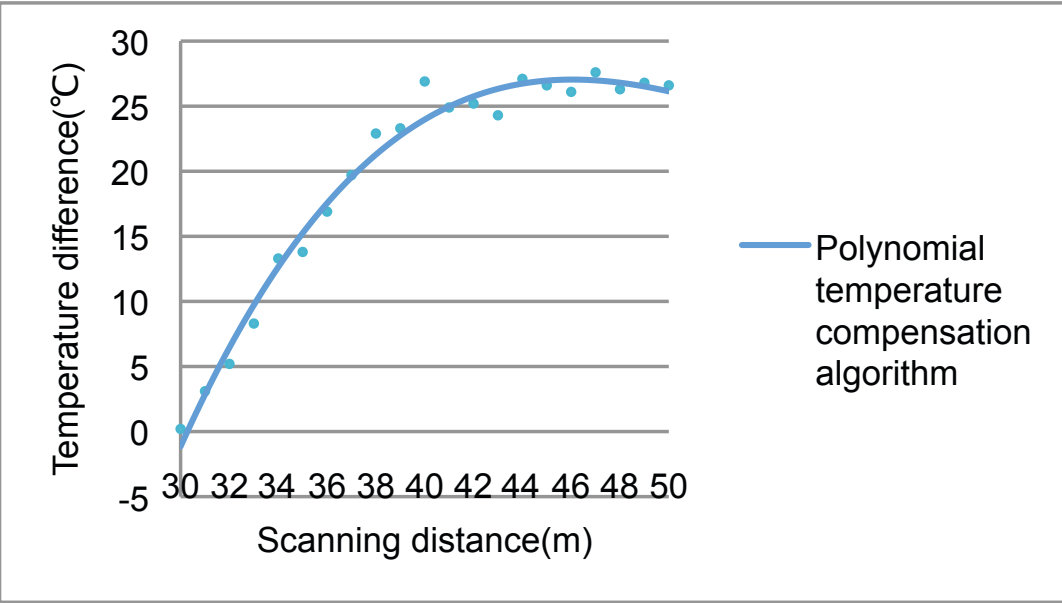

Figure.4 Temperature difference between real temperature and temperature value measured by ISTMS

The specific formula for the curve in the Fig. 4 is as below:

$$
\Delta T=0.0026 s^{3}-0.4218 s^{2}+22.57 s-367.71
$$

From the above experimental process, the realization process of nonlinear temperature compensation algorithm is summed up as follow, as shown in Figure.5:

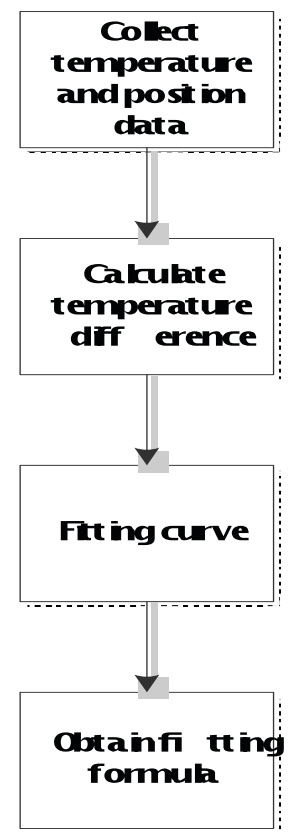

Fig.5 Realization process of nonlinear temperature compensation algorithm

1. Scanning points on rotary kiln surface are selected and the corresponding 
scanning distance between the points and ISTMS are recorded as $s_{1}, s_{2}, s_{3}, \ldots \ldots, s_{n}$, the temperature values of the scanning points are measured by ISTMS and handheld infrared thermometer respectively, which represent the temperature values before compensation and the real temperature values, these data are recorded as $T_{i_{1}}, T_{i_{2}}, T_{i_{3}}, \ldots \ldots, T_{i_{n}}$ and $T_{r_{1}}, T_{r_{2}}, T_{r_{3}}, \ldots \ldots . ., T_{r_{n}}$ respectively.

2. The temperature difference between the real temperature values and the temperature values measured by ISTMS are calculated as below:

$$
\Delta T=T_{r}-T_{i}
$$

Then the temperature difference data are recorded as $\Delta T_{1}, \Delta T_{2}, \Delta T_{3}, \ldots \ldots, \Delta T_{n}$.

3. According to the values of $\Delta T_{1}, \Delta T_{2}, \Delta T_{3}, \ldots \ldots, \Delta T_{n}$ and $s_{1}, s_{2}, s_{3}, \ldots \ldots, s_{n}$, the cubic polynomial fitting curve is obtained and then the algorithm is applied to the temperature measurement software of ISTMS.

In this way, the nonlinear temperature compensation formula can be got by measuring several characteristic points, and the more selected scanning points, the higher the temperature measurement accuracy.

\section{Algorithms comparison and error analysis}

The two algorithms are used to compensate the temperature value respectively, then they are used to analyze temperature compensation error. For linear temperature compensation algorithm, it supposes that when the scanning distance is 50 meter, the temperature difference achieves the maximum, whose value is $26.6^{\circ} \mathrm{C}$.

$$
T_{0}=\Delta T_{\max } / \Delta d_{\max }=1.33
$$

The temperature value of scanning points on rotary kiln surface is calculated as below:

$$
T_{l}=T_{i}+\Delta T=T_{i}+1.33(s-30)
$$

For nonlinear cubic polynomial temperature compensation algorithm, the temperature value of scanning points on rotary kiln surface is calculated as follow:

$$
T_{n}=T_{i}+\Delta T=T_{i}+0.0026 s^{3}-0.4218 s^{2}+22.57 s-367.71
$$

Then the temperature values of two algorithms are compared with the real temperature value, as shown in Fig.6. 


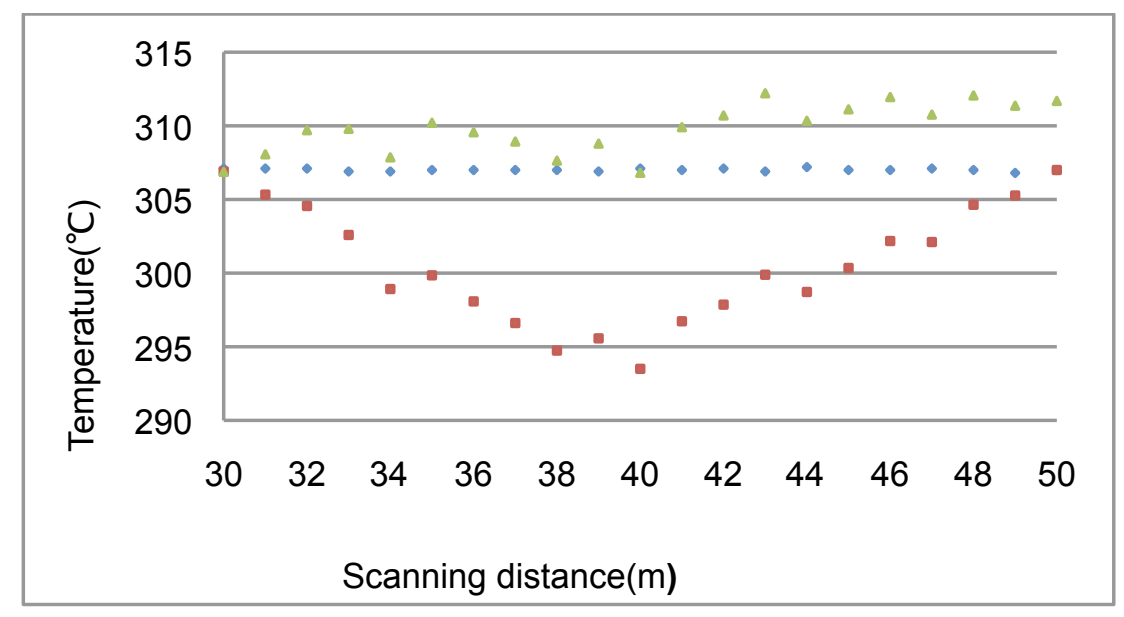

Fig.6 Compensated temperature values of two algorithms

Where the blue points represent the real temperature values, the green points represents the temperature values compensated by nonlinear cubic polynomial compensation algorithm, the red points represent linear compensation algorithm. From Fig. 6 it can be seen that compared with linear compensation algorithm, nonlinear temperature compensation algorithm is more in line with the temperature measurement demand. The temperature measurement errors of linear temperature compensation and nonlinear temperature compensation are shown in Fig.7.

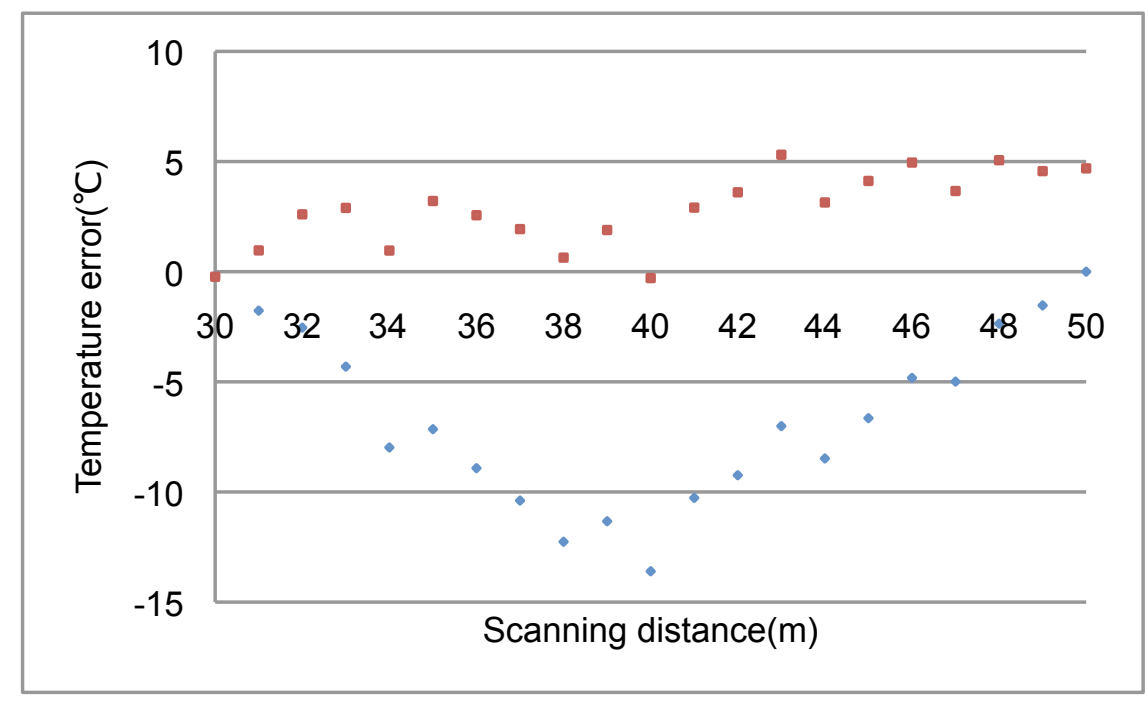

Fig.7 Temperature errors of two algorithms

The horizontal coordinate represents the scanning distance between ISTMS and black body radiator, and the vertical coordinate represents the temperature difference the display temperature and the temperature measured by ISTMS, the red points represent linear temperature compensation error, and the green points represent cubic polynomial fitting error.

For the linear temperature compensation algorithm, it supposes that the temperature error from 30 meter to 50 meter can be recorded as $E_{l_{1}}, E_{l_{2}}, E_{l_{3}}, \ldots, E_{l_{21}}$, the average error is represented as $E_{l a}$, it is calculated as below: 


$$
E_{l_{a}}=\frac{\sum_{n=1}^{21}\left|E_{l_{n}}\right|}{21} \approx 6.47
$$

For the nonlinear cubic polynomial temperature compensation algorithm, it supposes that the temperature error between 30 meter and 50 meter can be recorded as $E_{n_{1}}, E_{n_{2}}, E_{n_{3}}, \ldots, E_{n_{21}}$, the average error is represented as $E_{n_{a}}$, it is calculated as below:

$$
E_{n_{a}}=\frac{\sum_{n=1}^{21}\left|E_{n_{n}}\right|}{21} \approx 2.87
$$

Compared with the linear temperature compensation algorithm, the accuracy of nonlinear temperature compensation has raised about 2.25 times.

$$
E_{l_{a}} / E_{n_{a}} \approx 2.25
$$

\section{Conclusion}

This paper proposes a nonlinear cubic polynomial temperature compensation algorithm, which can effectively improve temperature measurement accuracy of rotary kiln, the proposed algorithm compensates the temperature value by selecting scanning points, calculating temperature difference, fitting curve and obtaining temperature compensation formula. Experiment result and analysis shows that compared with linear temperature compensation algorithm, the nonlinear temperature compensation algorithm not only has a higher temperature compensation accuracy, but also provides a new idea for temperature compensation of ISTMS.

\section{Acknowledgements}

This work is supported by the National Natural Science Foundation of China (No.61275099, No.61671094) and the National Science Foundation of Chongqing Science and Technology Commission (No.CSTC2015JCYJA40032).

\section{References}

[1] Dai S, Guo Z, You C, et al. Research on high precision equal-angle scanning method in rotary kiln temperature measurement system[J]. Infrared Physics \& Technology, 76:626-630(2016).

[2] Qiu L, Yao Q. Status Review and Development Trend Forecast of Rotary Kiln Temperature Measurement Technology [J]. Industrial Furnace, 36(6):16-20(2014).

[3] K.Chrzanowski, Influence of object-system distance on accuracy of remote temperature measuring with IR system, Infrared Physics \& Technology. 36(1995):703-713

[4] K.Chrzanowski, Influence of measurement conditions and system parameters on accuracy of remote temperature measurement with dual IR system, Infrared Physics \& Technology.36:703-713(1995) 
[5] Krapels K A, Driggers R G, Vollmerhausen R H, et al. Atmospheric effects in infrared target acquisition modeling[J]. Proceedings of SPIE - The International Society for Optical Engineering, 4372:182-1939(2001).

[6] Sun L, Kewei H, Xu D I, et al. Research on the Influence upon Accuracy of IR Thermal Temperature Measuring in Distance and Correction Method[J]. Journal of Changchun University of Science \& Technology, 31(1):33-35(2008).

[7] Guo B.H, Huang J.B, Analysis of affections on the accuracy of temperature measurement using infrared thermal imager system with the change of object distance and field, Journal of Changchun University of Science \& Technology,34:16-19(2011). [8] Zhang Y C, Chen Y M, Fu X B, et al. A method for reducing the influence of measuring distance on infrared thermal imager temperature measurement accuracy[J].Applied Thermal Engineering, 100:1095-1101(2016).

[9] Yi Z, Xiao H, Song J. An alumina rotary kiln monitoring system based on infrared ray scanning [J]. Measurement, 46(7):2051-2055(2013)

[10] Yang Bin. Performance of infrared scanning temperature measuring instrument and its application in rotary kiln [J], Cement, 7:40-43(2006)

[11] Zheng Z. Design of Infrared Thermometer with Wireless Data Transmission Function [J] . Electronic Technology, 38(11):56-58(2011).

[12] Zhu S, Lu R. Adopting Digital Filtering and Dynamic Compensation Technologies in PID Control [J]. Process Automation Instrumentation, 20(2): 11-12 (1999).

[13] Wang L L, Pan S L, Zi-Jian L U. Application of infrared ray scanning in monitoring of alumina rotational kiln[J]. Journal of Fushun Petroleum Institute, 23(4): 35-37(2003).

[14] LU Z, WANG X, PAN Y, et al. Influence of Object-system Distance on Accuracy of Temperature Measurement with IR System [J]. Infrared Technology, 30(5): 271-274 (2008) 